Homology, Homotopy and Applications, vol.13(1), 2011, pp.75-88

\title{
COLOCALIZATION FUNCTORS IN DERIVED CATEGORIES AND TORSION THEORIES
}

\author{
SHOHAM SHAMIR \\ (communicated by Luchezar Avramov)
}

\begin{abstract}
Let $R$ be a ring and let $\mathcal{A}$ be a hereditary torsion class of $R$-modules. The inclusion of the localizing subcategory generated by $\mathcal{A}$ into the derived category of $R$ has a right adjoint, denoted Cell $_{\mathcal{A}}$. Recently, Benson has shown how to compute Cell $_{\mathcal{A}} R$ when $R$ is a group ring of a finite group over a prime field and $\mathcal{A}$ is the hereditary torsion class generated by a simple module. We generalize Benson's construction to the case where $\mathcal{A}$ is any hereditary torsion class on $R$. It is shown that for every $R$-module $M$ there exists an injective $R$-module $E$ such that:
\end{abstract}

$$
H^{n}\left(\operatorname{Cell}_{\mathcal{A}} M\right) \cong \operatorname{Ext}_{\operatorname{End}_{R}(E)}^{n-1}\left(\operatorname{Hom}_{R}(M, E), E\right) \text { for } n \geqslant 2 \text {. }
$$

\section{Introduction}

Let $R$ be a ring and let $\mathcal{D}_{R}$ be the (unbounded) derived category of chain complexes of left $R$-modules. Fix a class $\mathcal{A}$ of objects of $\mathcal{D}_{R}$. We recall some definitions of Dwyer and Greenlees from [4]. An object $N$ of $\mathcal{D}_{R}$ is $\mathcal{A}$-null if $\operatorname{Ext}_{R}^{*}(A, N)=0$ for every $A \in \mathcal{A}$. An object $C$ of $\mathcal{D}_{R}$ is $\mathcal{A}$-cellular if $\operatorname{Ext}_{R}^{*}(C, N)=0$ for every $\mathcal{A}$-null $N$. An $\mathcal{A}$-cellular object $C$ is an $\mathcal{A}$-cellular approximation of $X \in \mathcal{D}_{R}$ if there is a map $\mu: C \rightarrow X$ such that $\operatorname{Ext}_{R}^{*}(A, \mu)$ is an isomorphism for all $A \in \mathcal{A}$. Finally, an $\mathcal{A}$-null object $N$ is an $\mathcal{A}$-nullification of $X$ if there is a map $\nu: X \rightarrow N$ which is universal among maps in $\mathcal{D}_{R}$ from $X$ to $\mathcal{A}$-null objects. Denote an $\mathcal{A}$-cellular approximation of $X$ by $\operatorname{Cell}_{\mathcal{A}} X$ and an $A$-nullification of $X$ by $\operatorname{Null}_{\mathcal{A}} X$.

The following properties are easy to check: A map $\mu: C \rightarrow X$ is an $\mathcal{A}$-cellular approximation of $X$ if and only if it is universal among all maps from $\mathcal{A}$-cellular objects to $X$. There is an exact triangle $\operatorname{Cell}_{\mathcal{A}} X \rightarrow X \rightarrow \operatorname{Null}_{\mathcal{A}} X$ whenever $\operatorname{Cell}_{\mathcal{A}} X$ or $\operatorname{Null}_{\mathcal{A}} X$ exists. An $\mathcal{A}$-cellular approximation of some object $X$ is unique up to isomorphism, and the same goes for an $\mathcal{A}$-nullification of $X$.

Now suppose $\mathcal{A}$ is a set; then it turns out that the full subcategory of $\mathcal{A}$-cellular objects is the localizing subcategory generated by $\mathcal{A}$ (see $[\mathbf{5}]$ and $[\mathbf{7}, 5.1 .5]$ ). Moreover, when $\mathcal{A}$ is a set, the inclusion functor of the full subcategory of $A$-cellular objects into $\mathcal{D}_{R}$ has a right adjoint, which is $\operatorname{Cell}_{\mathcal{A}} X$ for every $X \in \mathcal{D}_{R}$; see [6] or [7]. Hence

Received November 20, 2008, revised October 11, 2009, March 5, 2010, September 13, 2010; published on March 20, 2011.

2000 Mathematics Subject Classification: 16E30, 16S90.

Key words and phrases: torsion theory, colocalization, localization.

Article available at http://intlpress.com/HHA/v13/n1/a3 and doi:10.4310/HHA.2011.v13.n1.a3

Copyright (C) 2011, International Press. Permission to copy for private use granted. 
$\operatorname{Cell}_{\mathcal{A}}$ can be constructed as a colocalization functor (the right adjoint of an inclusion functor), and it follows that $\mathcal{A}$-cellular approximation and $\mathcal{A}$-nullification exist for any object of $\mathcal{D}_{R}$.

Similarly, when $\mathcal{A}$ is a set there exists a left adjoint to the inclusion of the full subcategory of $\mathcal{A}$-null objects; see, for example, Neeman's book [10, Section 9]. This functor is in fact $\mathcal{A}$-nullification and it is a localization functor (the left adjoint to an inclusion functor).

One method for calculating $\mathcal{A}$-cellular approximations is the formula given by Dwyer and Greenlees in [4], which holds whenever $\mathcal{A}=\{A\}$ and $A$ is a perfect complex. This was later generalized by Dwyer, Greenlees and Iyengar in [5]. A new method for calculating the $\mathcal{A}$-cellular approximation for $R$-modules has been constructed by Benson in [2], dubbed $k$-squeezed resolutions. This method can be applied whenever $\mathcal{A}$ is a set of simple modules and $R$ is an Artinian ring. One major benefit of Benson's construction is that it allows for explicit calculations.

As we will see, it is more natural to use Benson's method to construct the $\mathcal{A}$-nullification of a module rather than its $\mathcal{A}$-cellular approximation. We generalize Benson's construction so that it applies whenever $\mathcal{A}$ is a hereditary torsion class of modules. A hereditary torsion class of modules is a class of modules that is closed under submodules, quotient modules, coproducts and extensions. The main result of this paper is the following:

Theorem 1.1. Let $\mathcal{T}$ be a hereditary torsion class on left $R$-modules. For every left $R$-module $M$ there exists an injective left $R$-module $E$ such that the complex

$$
\mathbf{R H o m}_{E_{R}(E)}\left(\operatorname{Hom}_{R}(M, E), E\right)
$$

is a $\mathcal{T}$-nullification of $M$. In particular, the $\mathcal{T}$-nullification of $R$ is the differential graded algebra $\mathbf{R E n d}_{\operatorname{End}_{R}(E)}(E)$.

The formula given in the abstract follows immediately from the distinguished triangle $\mathrm{Cell}_{\mathcal{T}} M \rightarrow M \rightarrow \operatorname{Null}_{\mathcal{T}} M$ mentioned above.

The layout of this paper is as follows: The necessary background on hereditary torsion classes and the background on cellular approximations and nullifications is given in Section 2. In Section 3 we describe the construction of nullification with respect to a hereditary torsion class and prove Theorem 1.1. We study the case where $R$ is an Artinian ring in Section 4. This section offers a different proof to a result of Benson ([2, Theorem 5.1]). Finally, Section 5 provides several examples.

\subsection{Notation and terminology}

By a ring we always mean an associative ring with a unit, not necessarily commutative. Unless otherwise noted all modules considered are left modules. A triangle always means an exact (distinguished) triangle in the unbounded derived category of left $R$-modules, denoted $\mathcal{D}_{R}$. A complex is always a chain-complex of $R$-modules. For complexes we use the standard convention that the subscript grading is the negative of the superscript grading; i.e., $\square_{-i}=\square^{i}$. It is taken for granted that every $R$-module is a complex concentrated in degree 0 and with zero differential. A complex $X$ is bounded-above if, for some $n$ and for all $i>n, H_{i}(X)=0$. For complexes $X$ and $Y$ the notation $\operatorname{Hom}_{R}(X, Y)$ stands for the usual chain complex of homomorphisms. The notation $\mathbf{R H o m}_{R}(-,-)$ stands for the derived functor of the $\operatorname{Hom}_{R}(-,-)$ functor. 
By $\operatorname{End}_{R}(M)$ we mean the endomorphisms ring of an $R$-module $M$. The symbol $\simeq$ stands for quasi-isomorphism of complexes.

\section{Acknowledgements}

I am grateful to D. J. Benson for an illuminating explanation of his construction.

\section{Background on hereditary torsion theories and cellular- approximation, nullification and completion}

\subsection{Hereditary torsion theories}

Below is a recollection of the definition and main properties of hereditary torsion theories. A thorough review of this material can be found in [11].

Definition 2.1. A hereditary torsion class $\mathcal{T}$ is a class of $R$-modules that is closed under submodules, quotient modules, coproducts and extensions. Closure under extensions means that if $0 \rightarrow M_{1} \rightarrow M_{2} \rightarrow M_{3} \rightarrow 0$ is a short exact sequence with $M_{1}$ and $M_{3}$ in $\mathcal{T}$, then so is $M_{2}$. The modules in $\mathcal{T}$ will be called $\mathcal{T}$-torsion modules (or just torsion modules when the torsion theory is clear from the context). The class of torsion-free modules $\mathcal{F}$ is the class of all modules $F$ satisfying $\operatorname{Hom}_{R}(C, F)=0$ for every $C \in \mathcal{T}$. The pair $(\mathcal{T}, \mathcal{F})$ is referred to as a hereditary torsion theory. To every hereditary torsion theory there is an associated radical $t$, where $t(M)$ is the maximal torsion submodule of $M$. Note that $M / t(M)$ is therefore torsion-free.

Every hereditary torsion class $\mathcal{T}$ has an injective cogenerator (see [11, VI.3.7]). This means there exists an injective module $E$ such that a module $M$ is torsion if and only if $\operatorname{Hom}_{R}(M, E)=0$. It is also important to note that in any hereditary torsion theory, the class of torsion-free modules is closed under injective hulls (see [11, VI.3.2]). Thus, if $F$ is a torsion-free module then the injective hull of $F$ is also torsionfree.

Definition 2.2. Let $(\mathcal{T}, \mathcal{F})$ be a hereditary torsion theory and let $t$ be the associated radical. An $R$-module $M$ is called $\mathcal{F}$-closed if, for every left ideal $\mathfrak{a} \subset R$ such that $R / \mathfrak{a} \in \mathcal{T}$, the induced map $M=\operatorname{Hom}_{R}(R, M) \rightarrow \operatorname{Hom}_{R}(\mathfrak{a}, M)$ is an isomorphism. The inclusion of the full subcategory of $\mathcal{F}$-closed modules has a left adjoint $M \mapsto M_{\mathcal{F}}$. The module $M_{\mathcal{F}}$ is called the module of quotients of $M$ (see [11, IX.1]). The unit of this adjunction has the following properties: the kernel of the map $M \rightarrow M_{\mathcal{F}}$ is $t(M)$, $M_{\mathcal{F}}$ is torsion-free and the cokernel of this map is a torsion module.

\subsection{Cellular-approximation, nullification and completion}

The following recalls the basic properties of cellular approximation, as well as the definition of completion given by Dwyer and Greenlees in [4]:

Definition 2.3. Let $R$ be a ring and let $\mathcal{A}$ be a class of $R$-complexes. We say that an $R$-complex $X$ is $\mathcal{A}$-complete if $\operatorname{Ext}_{R}^{*}(N, X)=0$ for any $\mathcal{A}$-null object $N$. An $R$-complex $C$ is an $\mathcal{A}$-completion of $X$ if $C$ is $\mathcal{A}$-complete and there is an $\mathcal{A}$-equivalence $X \rightarrow C$. It is easy to see that an $\mathcal{A}$-completion of a complex $X$ is unique up to an isomorphism in $\mathcal{D}_{R}$. As in [4], we denote an $\mathcal{A}$-completion of $X$ by $X_{\mathcal{A}}^{\wedge}$. 
The following criterion for nullification is usually easier to check than the original definition. Its proof is easy and therefore omitted.

Lemma 2.4. Let $R$ be a ring and let $\mathcal{A}$ be a class of $R$-complexes. $A$ complex $N$ is an $\mathcal{A}$-nullification of $X$ if there is a triangle $C \rightarrow X \rightarrow N$ such that $C$ is $\mathcal{A}$-cellular and $N$ is $\mathcal{A}$-null. In this case it also follows that $C$ is an $A$-cellular approximation of $X$.

Recall that when $\mathcal{A}$ is a set, the full subcategory of $\mathcal{A}$-cellular objects in $\mathcal{D}_{R}$ is the localizing subcategory generated by $A$. The localizing category generated by $\mathcal{A}$, denoted $\langle\mathcal{A}\rangle$, is the smallest full triangulated subcategory of $\mathcal{D}_{R}$ that is closed under triangles, direct sums and retracts. Closure under triangles means that for every distinguished triangle in $\mathcal{D}_{R}$, if two of the objects are in the localizing subcategory, then so is the third. The proof of the following lemma is clear.

Lemma 2.5. Let $\mathcal{A}$ be a class of $R$-complexes; then every object of $\langle\mathcal{A}\rangle$ is $\mathcal{A}$-cellular. If $\mathcal{B}$ is another class of $R$-complexes such that $\langle\mathcal{A}\rangle=\langle\mathcal{B}\rangle$, then $\mathcal{A}$-cellular approximation is the same as $\mathcal{B}$-cellular approximation.

Remark 2.6. In [4] $\mathcal{A}$-cellular complexes were called $\mathcal{A}$-torsion while the term $\mathcal{A}$-cellular was reserved for complexes in $\langle\mathcal{A}\rangle$. When $\mathcal{T}$ is a hereditary torsion theory, the two terms agree (by Lemma 2.8 below).

\subsection{Cellular-approximation with respect to a hereditary torsion theory}

Let $\mathcal{T}$ be a hereditary torsion class. It is not immediately apparent that $\mathcal{T}$-cellular approximation exists. Below, in Lemma 2.8, we show that $\langle\mathcal{T}\rangle$ is the same as the localizing subcategory generated by a set $\mathcal{A}_{\mathcal{T}}$. This immediately implies that $\mathcal{T}$-cellular approximation and $\mathcal{T}$-nullification exist for any $R$-complex; see Corollary 2.9.

Definition 2.7. Given a hereditary torsion class of $R$-modules $\mathcal{T}$, we denote by $\mathcal{A}_{\mathcal{T}}$ the set of all cyclic $\mathcal{T}$-torsion modules.

Lemma 2.8. Let $\mathcal{T}$ be a hereditary torsion class; then every $\mathcal{T}$-torsion module is $\mathcal{A}_{\mathcal{T}}$-cellular and hence $\langle\mathcal{T}\rangle=\left\langle\mathcal{A}_{\mathcal{T}}\right\rangle$.

Proof. Clearly, every cyclic $\mathcal{T}$-torsion module is $\mathcal{A}_{\mathcal{T}}$-cellular. Therefore every direct sum of cyclic $\mathcal{T}$-torsion modules is $\mathcal{A}_{\mathcal{T}}$-cellular. Let $M$ be a $\mathcal{T}$-torsion module; then there is a surjection $C(M)=\oplus_{m \in M} R / \operatorname{ann}(m) \rightarrow M$. Since every hereditary torsion theory is closed under submodules, $R / \operatorname{ann}(m)$ is $\mathcal{T}$-torsion for every $m \in M$. Clearly,

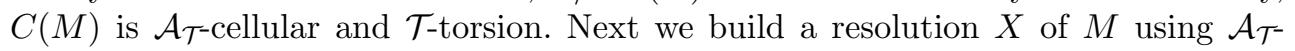
cellular modules. Let $X_{0}=C(M)$ and let $d_{0}: X_{0} \rightarrow M$ be the map defined above. The kernel of $d_{0}$ is $\mathcal{T}$-torsion, so there is an epimorphism $C\left(\operatorname{ker}\left(d_{0}\right)\right) \rightarrow \operatorname{ker}\left(d_{0}\right)$. Let $X_{1}=C\left(\operatorname{ker}\left(d_{0}\right)\right)$ and let $d_{1}$ be the composition $X_{1} \rightarrow \operatorname{ker}\left(d_{0}\right) \hookrightarrow X_{0}$. In this way $X$ is built inductively, and it is clear that $X$ is quasi-isomorphic to $M$. By construction, $X$ is in the localizing subcategory generated by $\mathcal{A}_{\mathcal{T}}$.

Corollary 2.9. Let $\mathcal{T}$ be a hereditary torsion class; then $\mathcal{T}$-cellular approximation, $\mathcal{T}$-nullification and $\mathcal{T}$-completion exist for every complex. Moreover, a complex $X$ is $\mathcal{T}$-cellular if and only if $X \in\langle\mathcal{T}\rangle$. 
Proof. Lemma 2.8 implies that $\mathcal{T}$-cellular approximation is the same as $\mathcal{A}_{\mathcal{T}}$-cellular approximation. As mentioned in Section $1, \mathcal{A}_{\mathcal{T}}$-cellular approximation exists for every complex. The proof of the other claims is similar.

Lemma 2.10. Let $\mathcal{T}$ be a hereditary torsion class.

1. If $X$ is a $\mathcal{T}$-cellular complex, then the homology groups of $X$ are $\mathcal{T}$-torsion $R$-modules.

2. If $X$ is a bounded-above complex such that the homology groups of $X$ are $\mathcal{T}$-torsion, then $X$ is $\mathcal{T}$-cellular.

Proof. Let $\mathcal{C}$ be the full subcategory of $\mathcal{D}_{R}$ containing all objects whose homology groups are $\mathcal{T}$-torsion $R$-modules. The properties of a hereditary torsion theory show that $\mathcal{C}$ is a localizing subcategory. Since $\mathcal{C}$ contains $\mathcal{T}$, then $\mathcal{C}$ also contains $\langle\mathcal{T}\rangle$. This proves the first statement.

Now suppose $X$ is a bounded-above complex and that $H_{i} X \in \mathcal{T}$ for all $i$. Because $X$ is bounded-above, $X$ belongs to the localizing subcategory generated by the homology groups of $X$ (see for example $[4,5.2]$ ). Since the homology groups of $X$ all belong to $\langle\mathcal{T}\rangle$, so does $X$.

Remark 2.11. If $R$ is a commutative Noetherian ring, then a complex $X$ is $\mathcal{T}$-cellular if and only if all the homology groups of $X$ are $\mathcal{T}$-torsion. This easily follows from a result of Neeman [9, Theorem 2.8]. However, Example 5.2 shows a noncommutative ring $R$ and a complex $X$ such that $H_{i}(X)$ is $\mathcal{T}$-torsion for all $i$, but $X$ is not $\mathcal{T}$-cellular.

\section{Nullification construction}

In [2] Benson gives a construction called $k$-squeezed resolution which yields $k$-cellular approximations over the $\operatorname{ring} k G$, where $k$ is a prime field and $G$ is a finite group. In Construction 3.1 we generalize Benson's work so as to produce $\mathcal{T}$-cellular approximations over any $\operatorname{ring} R$, where $\mathcal{T}$ is a hereditary torsion class. A second isomorphic construction of $k$-cellular approximations is given in Construction 3.2.

Nullification Construction 3.1. Let $(\mathcal{T}, \mathcal{F})$ be a hereditary torsion theory with radical $t$. For an $R$-module $M$ we construct the $\mathcal{T}$-nullification of $M$ as a cochain complex $I^{0} \stackrel{d}{\rightarrow} I^{1} \stackrel{d}{\rightarrow} I^{2} \stackrel{d}{\rightarrow} \cdots$ inductively.

Let $M^{0}=M$, let $F^{0}=M^{0} / t\left(M^{0}\right)$ and let $I^{0}$ be the injective hull of $F^{0}$. Note that since $F^{0}$ is torsion-free, so is $I^{0}$. We proceed by induction; set

$$
M^{n+1}=I^{n} / F^{n}, \quad F^{n+1}=M^{n+1} / t\left(M^{n+1}\right)
$$

and let $I^{n+1}$ be the injective hull of $F^{n+1}$. Again $I^{n+1}$ is torsion-free because $F^{n+1}$ is. The differential $d: I^{n} \rightarrow I^{n+1}$ is the composition $I^{n} \rightarrow M^{n+1} \rightarrow F^{n+1} \rightarrow I^{n+1}$. The image of $d: I^{n} \rightarrow I^{n+1}$ is $F^{n+1}$, and therefore $d \circ d=0$. Denote the resulting complex by $I$. The natural map $M \rightarrow I^{0}$ extends to a map of complexes $M \rightarrow I$.

Nullification Construction 3.2. For an $R$-module $M$ we construct a cochain complex $J^{0} \stackrel{d^{0}}{\rightarrow} J^{1} \stackrel{d^{1}}{\longrightarrow} J^{2} \stackrel{d^{2}}{\rightarrow} \cdots$ inductively. 
Let $Q^{0}=M$, let $N^{0}=\left(Q^{0}\right)_{\mathcal{F}}$ and let $J^{0}$ be the injective hull of $N^{0}$. Denote by $d^{-1}$ the map $M \rightarrow J^{0}$. Now proceed by induction; let

$$
Q^{n+1}=J^{n} / \operatorname{im}\left(d^{n-1}\right), \quad N^{n+1}=\left(Q^{n+1}\right)_{\mathcal{F}}
$$

and let $J^{n+1}$ be the injective hull of $N^{n+1}$. The differential $d^{n}: J^{n} \rightarrow J^{n+1}$ is the composition $J^{n} \rightarrow Q^{n+1} \rightarrow N^{n+1} \rightarrow J^{n+1}$. Clearly, $d^{n+1} \circ d^{n}=0$. Denote the resulting complex by $J$. The natural map $M \rightarrow J^{0}$ extends to a map of complexes $M \rightarrow J$. Note that for every $n, J^{n}$ is torsion-free because $N^{n}$ is.

Remark 3.3. For a fixed $R$-module $M$, the complex $I$ of Construction 3.1 and the complex $J$ of Construction 3.2 are isomorphic. To construct this isomorphism one needs the following property: for any $R$-module $L$ the injective hull of $L_{\mathcal{F}}$ and the injective-hull of $L / t(L)$ are the same; this is because $(L / t(L))_{\mathcal{F}}=L_{\mathcal{F}}$ and $L / t(L)$ is an essential submodule of $(L / t(L))_{\mathcal{F}}$ (see [11, IX.2.4]). Using the aforementioned property, it is a simple exercise to construct the isomorphism inductively.

Lemma 3.4. Let $J$ be the complex constructed from $M$ in 3.2; then $H_{0}(J) \cong M_{\mathcal{F}}$.

Proof. It easily follows from the definition of an $\mathcal{F}$-closed module that any injective torsion-free module is $\mathcal{F}$-closed; therefore $J^{0}$ is $\mathcal{F}$-closed. For any $\mathcal{F}$-closed module $K$ there is an isomorphism $K \cong K_{\mathcal{F}}$ (see [11, page 198]); therefore $\left(J^{0}\right)_{\mathcal{F}} \cong J^{0}$ and $\left(M_{\mathcal{F}}\right)_{\mathcal{F}} \cong M_{\mathcal{F}}$.

The module of quotients functor is left exact (see [11, page 199]). Hence applying the module of quotients functor to the sequence $M_{\mathcal{F}} \rightarrow J^{0} \rightarrow J^{0} / M_{\mathcal{F}}$ yields an exact sequence:

$$
0 \rightarrow M_{\mathcal{F}} \rightarrow J^{0} \rightarrow\left(J^{0} / M_{\mathcal{F}}\right)_{\mathcal{F}}
$$

We see that $J^{0} / M_{\mathcal{F}}$ is torsion-free because it is isomorphic to a submodule of the torsion-free module $\left(J^{0} / M_{\mathcal{F}}\right)_{\mathcal{F}}$.

Now consider the short exact sequence

$$
M_{\mathcal{F}} / \operatorname{im}(M) \rightarrow Q^{1} \rightarrow J^{0} / M_{\mathcal{F}}
$$

The module $M_{\mathcal{F}} / \operatorname{im}(M)$ is a torsion module (see Definition 2.2), while the module $J^{0} / M_{\mathcal{F}}$ is torsion free. From the definition of the radical $t$ it follows that $M_{\mathcal{F}} / \operatorname{im}(M) \cong$ $t\left(Q^{1}\right)$. Therefore $M_{\mathcal{F}}$ is the kernel of $J^{0} \rightarrow N^{1}$ and the proof is complete.

Lemma 3.5. Let $M$ be an $R$-module, let $I$ be the complex constructed from $M$ in 3.1 and let $C$ be a complex such that $C \rightarrow M \rightarrow I$ is a distinguished triangle. Then $C$ is a $\mathcal{T}$-cellular approximation of $M$, and $I$ is a $\mathcal{T}$-nullification of $M$. In particular, $H_{0}\left(\operatorname{Null}_{\mathcal{T}} M\right) \cong M_{\mathcal{F}}$ for any $R$-module $M$.

Proof. We can choose $C$ to be the complex $M \rightarrow I^{0} \rightarrow I^{1} \rightarrow \cdots$ with $M$ in degree 0 . The homology of $C$ is easy to compute: $H^{n}(C)=t\left(M^{n}\right)$, with $M^{n}$ as defined in Construction 3.1 above. By Lemma 2.10, the complex $C$ is $\mathcal{T}$-cellular. The complex $I$ is $\mathcal{T}$-null, simply because $I$ is composed of torsion-free injective modules. Thus, by Lemma $2.4, I$ is a $\mathcal{T}$-nullification of $M$ and $C$ is a $\mathcal{T}$-cellular approximation of $M$. Since $I$ is isomorphic to the complex $J$ of Construction 3.2, it follows that $H_{0}\left(\mathrm{Null}_{\mathcal{T}} M\right)=$ $H_{0}(J) \cong M_{\mathcal{F}}$. 
Using the constructions above we can give a different description of $\mathcal{T}$-nullification, the one shown in Theorem 1.1. Before proving Theorem 1.1 it is necessary to note some properties of the functor $\operatorname{Hom}_{R}(-, E)$.

Let $E$ be an $R$-module and $\mathcal{E}$ be the endomorphism $\operatorname{ring} \operatorname{End}_{R}(E)=\operatorname{Hom}_{R}(E, E)$. The functor $\operatorname{Hom}_{R}(-, E)$ is a contravariant functor from left $R$-complexes to left $\mathcal{E}$-complexes. This left $\mathcal{E}$-action is simply composition on the left with the morphisms in $\mathcal{E}$. In other words, the left $\mathcal{E}$-action on $\operatorname{Hom}_{R}(-, E)$ is induced by the left $\mathcal{E}$-action on $E$ itself. Moreover, the functor $\operatorname{Hom}_{\mathcal{E}}(-, E)$ is a contravariant functor, this time from left $\mathcal{E}$-complexes to left $R$-complexes. Here the left $R$-action on $\operatorname{Hom}_{\mathcal{E}}(-, E)$ comes from the left $R$-action on $E$ (which commutes with the left $\mathcal{E}$-action on $E$ ). In particular, there is a derived version of this functor: $\mathbf{R H o m}_{\mathcal{E}}(-, E): \mathcal{D}_{\mathcal{E}} \rightarrow \mathcal{D}_{R}$.

Proof of Theorem 1.1. Given an $R$-module $M$, construct a $\mathcal{T}$-nullification of $M$ in the way prescribed in 3.1. This construction results in a cochain complex $I$, with $I^{n}$ being an injective torsion-free module. Let $E$ be a torsion-free injective $R$-module such that for every $n, I^{n}$ is a direct summand of a finite direct sum of copies of $E$. For example, one can take $E$ to be the product $\prod_{n} I^{n}$. Denote by $\mathcal{E}$ the endomorphism $\operatorname{ring} \operatorname{End}_{R}(E)$.

Now consider the triangle $C \rightarrow M \rightarrow I$. Since $I$ is a $\mathcal{T}$-nullification of $M, C$ is a $\mathcal{T}$-cellular approximation of $M$. Applying the functor $\operatorname{Hom}_{R}(-, E)$ to this triangle yields a triangle in $\mathcal{D}_{\mathcal{E}}$ :

$$
\operatorname{Hom}_{R}(I, E) \rightarrow \operatorname{Hom}_{R}(M, E) \rightarrow \operatorname{Hom}_{R}(C, E) .
$$

Since $E$ is injective, $H_{i}\left(\operatorname{Hom}_{R}(C, E)\right) \cong \operatorname{Hom}_{R}\left(H_{i}(C), E\right)$. Since the homology groups of $C$ are torsion, $\operatorname{Hom}_{R}\left(H_{i}(C), E\right)=0$. Hence the $\operatorname{map}_{\operatorname{Hom}_{R}}(I, E) \rightarrow \operatorname{Hom}_{R}(M, E)$ is a quasi-isomorphism of $\mathcal{E}$-complexes.

Because $I^{n}$ is a direct summand of a finite direct sum of copies of $E$, the $\mathcal{E}$-module $\operatorname{Hom}_{R}\left(I^{n}, E\right)$ is projective. Thus the $\operatorname{map}_{\operatorname{Hom}}(I, E) \rightarrow \operatorname{Hom}_{R}(M, E)$ is a projective resolution of $\operatorname{Hom}_{R}(M, E)$ in the category of $\mathcal{E}$-modules. We conclude that the complex $\operatorname{Hom}_{\mathcal{E}}\left(\operatorname{Hom}_{R}(I, E), E\right)$ is the derived functor $\mathbf{R H o m}_{\mathcal{E}}\left(\operatorname{Hom}_{R}(M, E), E\right)$.

Because $I^{n}$ is a direct summand of a finite direct sum of copies of $E$, one readily sees that the $R$-module

$$
\operatorname{Hom}_{\mathcal{E}}\left(\operatorname{Hom}_{R}\left(I^{n}, E\right), E\right)
$$

is naturally isomorphic to $I^{n}$, and therefore $\operatorname{Hom}_{\mathcal{E}}\left(\operatorname{Hom}_{R}(I, E), E\right) \cong I$.

Remark 3.6. As noted in Theorem 1.1, $\operatorname{Null}_{\mathcal{T}} R \simeq \mathbf{R E n d}_{\mathcal{E}}(E)$, and therefore

$$
R_{\mathcal{F}} \cong H^{0}\left(\operatorname{Null}_{\mathcal{T}} R\right) \cong H^{0}\left(\mathbf{R E n d}_{\mathcal{E}}(E)\right)=\operatorname{End}_{\mathcal{E}}(E)
$$

This isomorphism recovers [11, IX.3.3], where it is stated that there is an injective $R$-module $E$ such that $R_{\mathcal{F}} \cong \operatorname{End}_{\operatorname{End}_{R}(E)}(E)$. Also note that $\operatorname{Null}_{\mathcal{T}} R$ is quasi-isomorphic to a differential graded algebra. This also follows from a result of Dwyer $[\mathbf{3}$, Proposition 2.5], where it is shown that for any set of complexes $\mathcal{A}$ the complex $\mathrm{Null}_{\mathcal{A}} R$ is quasi-isomorphic to a differential graded algebra.

Remark 3.7. Let $X=\cdots \rightarrow X_{n} \rightarrow X_{n-1} \rightarrow \cdots$ be a complex such that there exists some $m$ for which $X_{n}=0$ for all $n>m$. Then it is possible to generalize the Nullification Construction 3.1 to give the $\mathcal{T}$-nullification of $X$. Moreover, this generalized 
construction of $\operatorname{Null}_{\mathcal{T}} X$ can be done in such a way that for $n>m\left(\operatorname{Null}_{\mathcal{T}} X\right)_{n}=0$, while for $n \leqslant m\left(\operatorname{Null}_{\mathcal{T}} X\right)_{n}$ is a finite direct sum of torsion-free injective modules. Therefore $\left(\mathrm{Null}_{\mathcal{T}} X\right)_{n}$ is itself a torsion-free injective for $n \leqslant m$. Now it is easy to see that the proof of Theorem 1.1 works for $\operatorname{Null}_{\mathcal{T}} X$ as well and yields the same result. Namely, there exists an injective $R$-module $E$ such that

$$
\operatorname{Null}_{\mathcal{T}} X \simeq \mathbf{R H o m}_{E_{R}(E)}\left(\operatorname{Hom}_{R}(X, E), E\right) .
$$

Clearly, this result carries over to any bounded-above complex $X$.

Say an injective module $E$ is sufficient to compute the $\mathcal{T}$-nullification of $M$ if

$$
\operatorname{Null}_{\mathcal{T}} M \simeq \mathbf{R} H \operatorname{Hom}_{\mathcal{E}}\left(\operatorname{Hom}_{R}(M, E), E\right),
$$

where $\mathcal{E}=\operatorname{End}_{R}(M)$. Given an $R$-module $M$ one can use the proof of Theorem 1.1 to construct an injective module $E$ which is sufficient to compute the $\mathcal{T}$-nullification of $M$. However, there are other injective modules sufficient to compute the $\mathcal{T}$-nullification of $M$, as shown by the following proposition.

Proposition 3.8. Let $M$ be an $R$-module and let $E$ be an injective cogenerator of $\mathcal{T}$. Denote by $\mathcal{E}$ the ring $\operatorname{End}_{R}(E)$.

1. If the $\mathcal{E}$-module $\operatorname{Hom}_{R}(M, E)$ has a resolution composed of finitely generated projective modules in each degree, then $E$ is sufficient to compute the $\mathcal{T}$-nullification of $M$.

2. There exists an ordinal $\alpha$ such that the module $E^{\prime}=\prod_{i<\alpha} E$ is sufficient to compute the $\mathcal{T}$-nullification of $M$.

Proof. Let $P$ be a finitely generated projective $\mathcal{E}$-module; then it is easy to see that $\operatorname{Hom}_{R}\left(\operatorname{Hom}_{\mathcal{E}}(P, E), E\right)$ is naturally isomorphic to $P$. Now let $F$ be a projective resolution of $\operatorname{Hom}_{R}(M, E)$ over $\mathcal{E}$ and assume $F$ is composed of finitely generated projective modules in each degree. Then $\operatorname{Hom}_{R}\left(\operatorname{Hom}_{\mathcal{E}}(F, E), E\right)$ is naturally isomorphic to $F$.

The quasi-isomorphism $\eta: F \rightarrow \operatorname{Hom}_{R}(M, E)$ induces a map

$$
\operatorname{Hom}_{\mathcal{E}}\left(\operatorname{Hom}_{R}(M, E), E\right) \rightarrow \operatorname{Hom}_{\mathcal{E}}(F, E) .
$$

Composing with the natural map $M \rightarrow \operatorname{Hom}_{\mathcal{E}}\left(\operatorname{Hom}_{R}(M, E), E\right)$ yields a map $\mu: M \rightarrow$ $\operatorname{Hom}_{\mathcal{E}}(F, E)$. It is easy to see that $\operatorname{Hom}_{R}(\mu, E)$ is the quasi-isomorphism $\eta$.

Consider the triangle $C \rightarrow M \stackrel{\mu}{\rightarrow} \operatorname{Hom}_{\mathcal{E}}(F, E)$. Clearly $\operatorname{Hom}_{\mathcal{E}}(F, E)$ is $\mathcal{T}$-null. Since $\operatorname{Hom}_{R}(\mu, E)$ is a quasi-isomorphism, $\operatorname{Hom}_{R}(C, E)$ is quasi-isomorphic to zero. This implies $\operatorname{Hom}_{R}\left(H_{i}(C), E\right)=0$ for all $i$. Since $E$ is an injective cogenerator for $\mathcal{T}$, $H_{i}(C)$ is torsion for all $i$. Clearly, $C$ is bounded-above and so, by Lemma 2.10, $C$ is $\mathcal{T}$-cellular. We conclude that $\operatorname{Hom}_{\mathcal{E}}(F, E)$ is a $\mathcal{T}$-nullification of $M$, and $E$ is sufficient to compute the $\mathcal{T}$-nullification of $M$.

We now turn our attention to the second item in the proposition. By [11, VI.3.9], every torsion-free module has a monomorphism to some direct product of copies of $E$. In particular, every torsion-free injective is a isomorphic to a direct summand of some direct product of copies of $E$.

Let $I$ be the complex described in the Nullification Construction 3.1. Let $E^{\prime}$ be a direct product of copies of $E$ such that for every $n, I^{n}$ is isomorphic to a direct summand of $E^{\prime}$. Clearly, the $\operatorname{End}_{R}\left(E^{\prime}\right)$-complex $\operatorname{Hom}_{R}\left(I, E^{\prime}\right)$ is a projective resolution of 
$\operatorname{Hom}_{R}\left(M, E^{\prime}\right)$, which is composed of finitely generated projective modules in every degree. Hence $E^{\prime}$ is sufficient to compute the $\mathcal{T}$-nullification of $M$.

\section{Torsion theories and cellular approximation in Artinian rings}

Throughout this section $R$ is an Artinian ring and $\mathbb{S}$ is a set of non-isomorphic simple modules of $R$. Define a class $\mathcal{F}$ of $R$-modules by

$$
\mathcal{F}=\left\{F \mid \operatorname{Hom}_{R}(S, F)=0 \text { for all } S \in \mathbb{S}\right\}
$$

and define a class $\mathcal{T}$ by setting

$$
\mathcal{T}=\left\{M \mid \operatorname{Hom}_{R}(M, F)=0 \text { for all } F \in \mathcal{F}\right\} .
$$

By [11, VIII.3], the pair $(\mathcal{T}, \mathcal{F})$ forms a hereditary torsion theory (alternatively, one can easily deduce this from Lemma 4.3 below). Because $R$ is Artinian, every hereditary torsion theory of $R$-modules is generated by a set of simple modules (see [11, VIII]), so this context covers all hereditary torsion theories over $R$. In this section we give several results regarding $\mathcal{T}$-nullification. We also give a different proof for a result of Benson [2, Theorem 5.1] in Corollary 4.5.

Let $\Omega$ be the set of isomorphism classes of simple modules of $R$ and let $\mathbb{S}^{\prime}$ be the complement of $\mathbb{S}$ in $\Omega$. We denote by $E$ the product of the injective hulls of the simple modules in $\mathbb{S}^{\prime}$ and denote by $P$ the direct sum of the projective covers of those simple modules. We show that $E$ is an injective cogenerator of $\mathcal{T}$ and that being $\mathcal{T}$-cellular is the same as being $\mathbb{S}$-cellular.

Lemma 4.1. Let $C$ be a cyclic $R$-module such that $\operatorname{Hom}_{R}(C, E)=0$; then $C$ is S-cellular.

Proof. Since $R$ is Artinian, $C$ admits a composition series

$$
0=C_{0} \subset C_{1} \subset \cdots \subset C_{m}=C,
$$

where all the quotients $C_{i} / C_{i-1}$ are simple modules. We next show that

$$
C_{i} / C_{i-1} \in \mathbb{S} \text { for all } i \text {. }
$$

Suppose that for some $i, C_{i} / C_{i-1} \cong S^{\prime}$ for some $S^{\prime} \in \mathbb{S}^{\prime}$. Let $x \in C_{i} \backslash C_{i-1}$; then the cyclic module generated by $x$ has $S^{\prime}$ as a quotient. This implies that the submodule $R x$ of $C$ has a non-zero map to $E\left(S^{\prime}\right)$ - the injective hull of $S^{\prime}$. Clearly, such a map can be lifted to a non-zero map $C \rightarrow E$, in contradiction. Therefore $C_{i} / C_{i-1} \cong S$ for some $S \in \mathbb{S}$. Now a simple inductive argument on $i$ shows that $C_{i} \in\langle\mathbb{S}\rangle$ for every $i$, and hence $C$ is $\mathbb{S}$-cellular.

Corollary 4.2. A complex $X$ is $\mathcal{T}$-cellular if and only if $X$ is $\mathbb{S}$-cellular.

Proof. We need to show that $\langle\mathcal{T}\rangle=\langle\mathbb{S}\rangle$. Since $\mathbb{S} \subset \mathcal{T}$, we only need to show that $\mathcal{T} \subset\langle\mathbb{S}\rangle$. By Lemma 2.8 it is enough to show that every cyclic $R$-module is $\mathbb{S}$-cellular, but that is immediate from Lemma 4.1.

Lemma 4.3. The module $E$ is an injective cogenerator for $\mathcal{T}$. 
Proof. Let $\mathcal{U}$ be the class of modules $M$ such that $\operatorname{Hom}_{R}(M, E)=0$. Then $\mathcal{U}$ is a hereditary torsion theory. Because $\operatorname{Hom}_{R}\left(S, S^{\prime}\right)=0$ for every $S \in \mathbb{S}$ and $S^{\prime} \in \mathbb{S}^{\prime}$, we see that $\operatorname{Hom}_{R}\left(S, E\left(S^{\prime}\right)\right)=0$, where $E\left(S^{\prime}\right)$ is the injective envelope of $S^{\prime}$. Hence $E \in \mathcal{F}$, and therefore $\mathcal{U}$ contains $\mathcal{T}$.

Next, let $M$ be in $\mathcal{U}$. To show that $M$ is a $\mathcal{T}$-torsion module it is enough to show that every cyclic submodule of $M$ is a torsion module, because $M$ is a quotient of the direct sum of its cyclic submodules. So let $C$ be a cyclic submodule of $M$. Since $E$ is injective, it follows that $\operatorname{Hom}_{R}(C, E)=0$. By Lemma 4.1 $C$ is $\mathbb{S}$-cellular. Therefore $C$ is $\mathcal{T}$-cellular, and by Lemma $2.10 C$ is $\mathcal{T}$-torsion.

Lemma 4.4. For any complex $X, \operatorname{Ext}_{R}^{*}(P, X)=0$ if and only if $\operatorname{Ext}_{R}^{*}(X, E)=0$.

Proof. This is known when $X$ is a finitely generated $R$-module; see Benson's book [1, 1.7.6 and 1.7.7]. Now suppose $X$ is any $R$-module. Since $P$ is a finitely generated projective module, $\operatorname{Hom}_{R}(P, X)=0$ if and only if $\operatorname{Hom}_{R}\left(P, X^{\prime}\right)=0$ for every finitely generated submodule $X^{\prime}$ of $X$. Similarly, because $E$ is injective, $\operatorname{Hom}_{R}(X, E)=0$ if and only if $\operatorname{Hom}_{R}\left(X^{\prime}, E\right)=0$ for every finitely generated submodule $X^{\prime}$ of $X$. Hence the lemma holds for any $R$-module. Finally, let $X$ be any complex; then $\operatorname{Ext}_{R}^{*}(P, X)=$ $\operatorname{Hom}_{R}\left(P, H_{*}(X)\right)$. Similarly, $\operatorname{Ext}_{R}^{*}(X, E)=\operatorname{Hom}_{R}\left(H^{*}(X), E\right)$.

Corollary 4.5. For any $R$-module $M$, a $\mathcal{T}$-nullification of $M$ is also a $P$-completion of $M$ and is therefore given by

$$
\operatorname{Null}_{\mathcal{T}} M \simeq \mathbf{R H o m}_{E^{2} n_{R}(P)}\left(\operatorname{Hom}_{R}(P, R), \operatorname{Hom}_{R}(P, M)\right) .
$$

Proof. Consider the triangle $\operatorname{Cell}_{\mathcal{T}} M \rightarrow M \stackrel{\nu}{\rightarrow} \operatorname{Null}_{\mathcal{T}} M$. Lemma 4.3 implies that $E$ is $\mathcal{T}$-null, and therefore $\operatorname{Ext}_{R}^{*}\left(\operatorname{Cell}_{\mathcal{T}} M, E\right)=0$. By Lemma 4.4, $\operatorname{Cell}_{\mathcal{T}} M$ is $P$-null and $\nu$ is a $P$-equivalence.

It remains to show that $\operatorname{Null}_{\mathcal{T}} M$ is $P$-complete. Let $I$ be the $\mathcal{T}$-nullification of $M$ described in 3.1. The full subcategory of $P$-complete objects in $\mathcal{D}_{R}$ is closed under isomorphisms, completion of triangles, products and retracts. Denote this subcategory by $\mathcal{C}$. From Lemma 4.4, we see that $E \in \mathcal{C}$, and therefore every product of $E$ is also in $\mathcal{C}$. Lemma 4.3 and [11, VI.3.9] imply that every torsion-free module is a submodule of a product of copies of $E$. Since $I^{n}$ is injective, it is a direct summand of some product of copies of $E$, hence $I^{n}$ is also an object of $\mathcal{C}$.

Let $I(n)$ denote the cochain complex $I^{0} \rightarrow I^{1} \rightarrow \cdots \rightarrow I^{n}$. An inductive argument

shows that $I(n) \in \mathcal{C}$. There is a triangle $I \rightarrow \prod_{n} I(n) \stackrel{\phi-1}{\longrightarrow} \prod_{n} I(n)$, where the map $\phi$ is induced by the maps $I(n+1) \rightarrow I(n)$. Hence $I$ is $P$-complete.

By Dwyer and Greenlees [4, Theorem 2.1], the $P$-completion of an $R$-module $M$ is given by

$$
M_{P}^{\wedge} \simeq \mathbf{R H o m}_{\operatorname{End}_{R}(P)}\left(\operatorname{Hom}_{R}(P, R), \operatorname{Hom}_{R}(P, M)\right) .
$$

Corollary 4.5 above implies Benson's formula for $\mathcal{T}$-cellular approximation given in [2, Theorem 5.1]. This corollary also explains the connection between Benson's formula and Dwyer and Greenlees formula for P-completion from [4, Theorem 2.1].

\section{Examples}

Example 5.1. Let $I$ be a two-sided ideal of $R$ such that $I$ is finitely generated as a left $R$-module. An $R$-module $M$ will be called $I$-torsion if for every $m \in M$ there 
exists some $n$ such that $I^{n} m=0$. It is not difficult to show that the class of $I$-torsion modules forms a hereditary torsion class $\mathcal{T}$ (see [11, VI.6.10]). Using Lemma 2.8 it is easy to conclude that $\langle\mathcal{T}\rangle=\langle R / I\rangle$. Hence $\mathcal{T}$-cellular approximation is the same as $R / I$-cellular approximation and the same goes for nullification. Note that in this case the radical $t$ associated with $\mathcal{T}$ has a simple description: for any $R$-module $M$

$$
t(M)=\operatorname{colim}_{n \rightarrow \infty} \operatorname{Hom}_{R}\left(R / I^{n}, M\right) .
$$

Now suppose $R$ is a commutative Noetherian ring. Dwyer and Greenlees have shown in [4] that $R / I$-cellular approximation computes $I$-local cohomology, namely that there is a natural isomorphism $H_{I}^{*}(M) \cong H^{*}\left(\operatorname{Cell}_{R / I} M\right)$. Recall there is an isomorphism:

$$
H_{I}^{*}(M) \cong \operatorname{colim}_{n \rightarrow \infty} \operatorname{Ext}_{R}^{*}\left(R / I^{n}, M\right)
$$

These facts show that $\mathcal{T}$-cellular approximation is the derived functor of the radical $t$. Moreover, in this case an object $X \in \mathcal{D}_{R}$ is $\mathcal{T}$-cellular if and only $H_{n}(X)$ is $\mathcal{T}$-torsion for all $n$; see $[4,6.12]$.

Example 5.2. Here is an example of a case where $\mathcal{T}$-cellular approximation is not the derived functor of the associated radical. Let $G$ be the symmetric group on three elements, let $k$ be the field $\mathbb{Z} / 3 \mathbb{Z}$ and let $R$ be the group ring $k[G]$. There is an augmentation map $R \rightarrow k$, where $k$ has the trivial $G$-action. Let $I$ be the augmentation ideal. As before, denote the class of $I$-torsion modules by $\mathcal{T}$ and the associated radical by $t$. Since $R$ is an Artinian ring, the sequence $I \supseteq I^{2} \supseteq I^{3} \supseteq \cdots$ stabilizes. So there is a fixed index $m$ such that $t(M)=\operatorname{Hom}_{R}\left(R / I^{m}, M\right)$ for every $R$-module $M$. Therefore, the derived functors of the torsion radical $t$ are the functors $\operatorname{Ext}_{R}^{*}\left(R / I^{m},-\right)$. In particular, $\operatorname{Ext}_{R}^{i}\left(R / I^{m}, R\right)=0$ for all $i>0$, because $R$ is injective. On the other hand, a calculation using Benson's methods from [2] shows that $H^{n}\left(\mathrm{Cell}_{\mathcal{T}} R\right)$ is nonzero for infinitely many values of $n$, thereby showing that $\mathrm{Cell}_{\mathcal{T}}$ is not the derived functor of $t$. We describe this calculation next.

From the surjection $G \rightarrow \mathbb{Z} / 2$ one sees that $R$ has two simple modules, the trivial module $k$ and a one dimensional simple module $\omega$. As a left module, $R \cong E_{k} \oplus E_{\omega}$ where $E_{k}$ and $E_{\omega}$ are the injective hulls of $k$ and $\omega$ respectively. The module $E_{k}$ has a composition series

$$
k \subset B \subset E_{k}, \quad \text { where } B / k \cong \omega \text { and } E_{k} / B \cong k .
$$

The composition series for $E_{\omega}$ is

$$
\omega \subset B^{\prime} \subset E_{\omega}, \quad \text { where } B^{\prime} / \omega \cong k \text { and } E_{\omega} / B^{\prime} \cong \omega .
$$

In addition, $E_{\omega} / \omega \cong B$ and $E_{k} / k \cong B^{\prime}$. Since $E_{\omega}$ is $k$-null (see Lemma 4.3 ), then

$$
\operatorname{Null}_{\mathcal{T}} R \simeq \operatorname{Null}_{\mathcal{T}} E_{\omega} \oplus \operatorname{Null}_{\mathcal{T}} E_{k} \cong E_{\omega} \oplus \operatorname{Null}_{\mathcal{T}} E_{k}
$$

So we need only compute $\mathrm{Null}_{\mathcal{T}} E_{k}$. Applying Construction 3.1 to the module $E_{k}$, we get the complex $I$ which is $E_{\omega} \stackrel{d}{\rightarrow} E_{\omega} \stackrel{d}{\rightarrow} E_{\omega} \stackrel{d}{\rightarrow} \cdots$, where $d$ is the composition 
$E_{\omega} \rightarrow \omega \hookrightarrow E_{\omega}$. Hence $H^{n}\left(\operatorname{Null}_{\mathcal{T}} E_{k}\right)=k$ for $n>1$, and therefore $H^{n}\left(\operatorname{Cell}_{\mathcal{T}} R\right)$ is nonzero for infinitely many values of $n$. In fact,

$$
H^{n}\left(\operatorname{Cell}_{\mathcal{T}} R\right)= \begin{cases}k, & n=0 \\ 0, & n=1 \\ k, & n>1\end{cases}
$$

It is important to note that, in this case, a complex $X$ such that $H_{n}(X)$ is $\mathcal{T}$ torsion for all $n$ need not be $\mathcal{T}$-cellular. Consider, for example, the complex $R_{\mathcal{T}}^{\wedge}$. As we explain below, the homology groups $H_{n}\left(R_{\mathcal{T}}^{\wedge}\right)$ are $\mathcal{T}$-torsion for all $n$. On the other

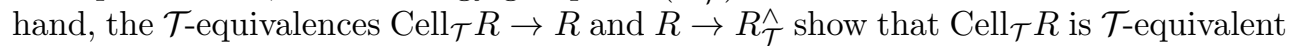
to $R_{\mathcal{T}}^{\wedge}$. If $R_{\mathcal{T}}^{\wedge}$ was $\mathcal{T}$-cellular, then $R_{\mathcal{T}}^{\wedge}$ would have been quasi-isomorphic to $\operatorname{Cell}_{\mathcal{T}} R$, because a $\mathcal{T}$-equivalence between $\mathcal{T}$-cellular complexes is a quasi-isomorphism. As we show next, $H^{n}\left(R_{\mathcal{T}}^{\wedge}\right)=0$ for $n>0$ and so $R_{\mathcal{T}}^{\wedge}$ cannot be quasi-isomorphic to $\operatorname{Cell}_{\mathcal{T}} R$.

It remains to explain the properties of $R_{\mathcal{T}}$ used above. From Corollary 4.2 we learn that $R_{\mathcal{T}}^{\wedge} \simeq R_{k}^{\wedge}$ and $\operatorname{Cell}_{\mathcal{T}} R \simeq \operatorname{Cell}_{k} R$. Without going into details, combining [5, 5.9] with $[\mathbf{4}, 4.3]$ shows that

$$
R_{k}^{\wedge} \simeq \mathbf{R H o m}_{R}\left(\operatorname{Cell}_{k} R, R\right) .
$$

This immediately implies that $H^{n}\left(R_{\mathcal{T}}^{\wedge}\right)=0$ for $n>0$. We next show that $H_{n}\left(R_{\mathcal{T}}^{\wedge}\right)$ is $\mathcal{T}$-torsion for all $n$. Since $E_{\omega}$ is a $\mathcal{T}$-null module, $\operatorname{Ext}_{R}^{*}\left(E_{\omega}, R_{\mathcal{T}}\right)=0$. Recall that $R$ is a group-algebra, and therefore $E_{\omega}$ is also the projective cover of $\omega$. Because $E_{\omega}$ is projective we have

$$
\operatorname{Ext}_{R}^{-n}\left(E_{\omega}, R_{\mathcal{T}}^{\wedge}\right) \cong \operatorname{Hom}_{R}\left(E_{\omega}, H_{n}\left(R_{\mathcal{T}}^{\wedge}\right)\right) .
$$

Hence, by Lemma $4.4, \operatorname{Ext}_{R}^{*}\left(H_{n}\left(R_{\mathcal{T}}^{\wedge}\right), E_{\omega}\right)=0$. Lemma 4.3 shows $E_{\omega}$ is an injective cogenerator for $\mathcal{T}$; therefore $H_{n}\left(R_{\mathcal{T}}^{\wedge}\right)$ is $\mathcal{T}$-torsion.

Example 5.3. This example relates $\mathcal{T}$-nullification with Cohn localization. We begin by recalling the definition of Cohn localization. Let $S=\left\{f_{\alpha}: P_{\alpha} \rightarrow Q_{\alpha}\right\}$ be a set of maps between finitely generated projective $R$-modules. Say a ring map $R \rightarrow R^{\prime}$ is $S$-inverting if $\operatorname{Hom}_{R}\left(f, R^{\prime}\right)$ is an isomorphism for every $f \in S$. A Cohn localization of $R$ with respect to $S$ is a ring map $R \rightarrow S^{-1} R$, which is initial among all $S$-inverting ring maps. Note that the definition given here is not the standard definition (see e.g. [3]), but it is equivalent to the standard one.

Let $\mathcal{C}_{S}$ be the set of cones of the maps $f_{\alpha}$. In $[\mathbf{3}]$, Dwyer considers $\mathcal{C}_{S^{-} \text {-nullification }}$ and shows that $H_{0}\left(\operatorname{Null}_{\mathcal{C}_{S}} R\right)=S^{-1} R$ (see [3, 3.2]). Combining Dwyer's results with Theorem 1.1 yields the following proposition.

Proposition 5.4. Let $\mathcal{T}$ be a hereditary torsion-class of R-modules. If $\langle\mathcal{T}\rangle=\left\langle\mathcal{C}_{S}\right\rangle$ for some set of maps $S$ between finitely generated projective $R$-modules, then

1. $\operatorname{Null}_{\mathcal{T}}(-) \simeq(-)_{\mathcal{F}}$,

2. the module of quotients functor $(-)_{\mathcal{F}}$ is exact and

3. there is an isomorphism $S^{-1} R \otimes_{R} M \cong M_{\mathcal{F}}$ for every module $M$.

Proof. By Theorem 1.1, for every $R$-module $M$ the complex $\operatorname{Null}_{\mathcal{T}} M$ has no homology in positive degrees. By [3, Proposition 3.1], $\operatorname{Null}_{\mathcal{T}} R$ has no homology in negative degrees. Moreover, a result of Miller [8] (see also [3, Proposition 2.10]) shows that 
for every $R$-module $M, \operatorname{Null}_{\mathcal{T}} M \simeq \operatorname{Null}_{\mathcal{T}} R \otimes_{R}^{\mathbf{L}} M$. This implies that $\mathrm{Null}_{\mathcal{T}} M$ has no homology in negative degrees.

We conclude that for every $R$-module $M, \mathrm{Null}_{\mathcal{T}} M$ has homology only in degree 0 , and therefore, by Lemma 3.5, $\operatorname{Null}_{\mathcal{T}} M$ is quasi-isomorphic to $M_{\mathcal{F}}$. Since the functor $\mathrm{Null}_{\mathcal{T}}$ is exact, so is $(-)_{\mathcal{F}}$. Since $\mathrm{Null}_{\mathcal{T}} R \simeq \operatorname{Null}_{\mathcal{C}_{S}} R$, Dwyer's result [3, 3.2] shows that $R_{\mathcal{F}} \cong S^{-1} R$. Finally, the quasi-isomorphism $\operatorname{Null}_{\mathcal{T}} M \simeq \operatorname{Null}_{\mathcal{T}} R \otimes_{R}^{\mathrm{L}} M$ implies $S^{-1} R \otimes_{R} M \cong M_{\mathcal{F}}$.

Example 5.5. This example is of a topological nature. Let $M$ be a discrete monoid and let $k=\mathbb{Z} / p \mathbb{Z}$ for some prime $p$. The ring $R$ we consider is the monoid $\operatorname{ring} R=k[M]$; it has a natural augmentation $R \rightarrow k$ with augmentation ideal $I$. We also make the following assumptions:

1. The classifying space $\mathrm{B} M$ of $M$ has a finite fundamental group.

2. The augmentation ideal $I$ is finitely generated as a left $R$-module.

3. There is a projective resolution $P=\cdots P_{2} \rightarrow P_{1} \rightarrow P_{0}$ of $k$ over $R$ such that every $P_{n}$ is finitely generated as an $R$-module.

Let $\mathcal{T}$ be the hereditary torsion class of $I$-torsion $R$-modules; then $\langle\mathcal{T}\rangle=\langle k\rangle$ and hence $\operatorname{Cell}_{\mathcal{T}} \simeq \mathrm{Cell}_{k}$. Denote by $R^{\vee}$ the left $R$-module $\operatorname{Hom}_{k}(R, k)$. From the results of Dwyer, Greenlees and Iyengar [5, 6.15 and 7.5], it is easy to conclude that $\mathrm{Cell}_{k} R^{\vee}$ is quasi-isomorphic to the cochain complex (with coefficients in $k$ ) of a certain space, which we describe next. Let $(\mathrm{B} M)_{p}^{\wedge}$ be the Bousfield-Kan $p$-completion of the classifying space of $M$. The space $\Omega(\mathrm{B} M)_{p}^{\wedge}$ is the loop-space of $(\mathrm{B} M)_{p}^{\wedge}$. So, $\operatorname{Cell}_{k} R^{\vee}$ is quasi-isomorphic to $\mathrm{C}^{*}\left(\Omega(\mathrm{B} M)_{p}^{\wedge} ; k\right)$ - the singular cochain complex of $\Omega(\mathrm{B} M)_{p}^{\wedge}$ with coefficients in $k$. By Theorem 1.1, there exists an injective $R$-module $E$ such that

$$
H^{n}\left(\Omega(\mathrm{B} M)_{p}^{\wedge} ; k\right) \cong \operatorname{Ext}_{\operatorname{End}_{R}(E)}^{n-1}\left(\operatorname{Hom}_{R}\left(R^{\vee}, E\right), E\right) \text { for } n \geqslant 2
$$

\section{References}

[1] D. J. Benson, Representations and cohomology. I. Basic representation theory of finite groups and associative algebras, Cambridge Studies in Advanced Mathematics 30, Cambridge University Press, Cambridge, 1991.

[2] D. J. Benson, An algebraic model for chains on $\Omega B G_{p}^{\wedge}$, Trans. Amer. Math. Soc. 361 (2009), no. 4, 2225-2242.

[3] W. G. Dwyer, Noncommutative localization in homotopy theory, in Noncommutative localization in algebra and topology, London Math. Soc. Lecture Note Ser. 330, Cambridge Univ. Press, Cambridge, 2006, pp 24-39.

[4] W. G. Dwyer and J. P. C. Greenlees, Complete modules and torsion modules, Amer. J. Math. 124 (2002), no. 1, 199-220.

[5] W. G. Dwyer, J. P. C. Greenlees and S. Iyengar, Duality in algebra and topology, Adv. Math. 200 (2006), no. 2, 357-402.

[6] E. D. Farjoun, Cellular spaces, null spaces and homotopy localization, Lecture Notes in Mathematics 1622, Springer-Verlag, New York, 1996.

[7] P. S. Hirschhorn, Model categories and their localizations, Mathematical Surveys and Monographs 99, Amer. Math. Soc., Providence, RI, 2003. 
[8] H. Miller, Finite localizations, Papers in honor of José Adem (Spanish), Bol. Soc. Mat. Mexicana (2) 37 (1992), no. 1-2, 383-389.

[9] A. Neeman, The chromatic tower for $D(R)$, With an appendix by Marcel Bökstedt, Topology 31 (1992), no. 3, 519-532.

[10] A. Neeman, Triangulated categories, Annals of Mathematics Studies 148, Princeton University Press, Princeton, NJ, 2001.

[11] B. Stenström, Rings of quotients. An introduction to methods of ring theory, Die Grundlehren der Mathematischen Wissenschaften 217, Springer-Verlag, New York, 1975.

Shoham Shamir shoham.shamir@math.uib.no

Department of Mathematics, University of Bergen, Johannes Brunsgate 12, P.O. Box 7803, 5008 Bergen, Norway 